\title{
Correction to: Biomedical Product Development: Bench to Bedside, Learning Materials in Biosciences
}

Babak Arjmand (D), Moloud Payab (D), and Parisa Goodarzi

Correction to: B. Arjmand et al. (eds.), Biomedical Product

Development: Bench to Bedside, Learning Materials in Biosciences, $>$ https://doi.org/10.1007/978-3-030-35626-2

The original version of the chapters 10 and 11 were inadvertently published with incorrect figures. Figure 1 of these chapters has now been updated.

The updated version of these chapters can be found at

https://doi.org/10.1007/978-3-030-35626-2_10

https://doi.org/10.1007/978-3-030-35626-2_11 